\title{
Article
}

\section{A scoping review of psychoeducational interventions for people after transient ischemic attack and minor stroke}

Kontou, E, Kettlewell, J, Condon, L, Thomas, S, Lee, AR, Sprigg, N, Watkins, Caroline Leigh, Walker, M and Shokraneh, F

Available at http://clok.uclan.ac.uk/34949/

Kontou, E, Kettlewell, J, Condon, L, Thomas, S, Lee, AR, Sprigg, N, Watkins, Caroline Leigh ORCID: 0000-0002-9403-3772, Walker, M and Shokraneh, F (2020) A scoping review of psychoeducational interventions for people after transient ischemic attack and minor stroke. Topics in Stroke Rehabilitation, 28 (5). pp. 390-400. ISSN 1074-9357

It is advisable to refer to the publisher's version if you intend to cite from the work. https://doi.org/10.1080/10749357.2020.1818473

For more information about UCLan's research in this area go to http://www.uclan.ac.uk/researchgroups/ and search for <name of research Group>.

For information about Research generally at UCLan please go to http://www.uclan.ac.uk/research/

All outputs in CLoK are protected by Intellectual Property Rights law, including Copyright law. Copyright, IPR and Moral Rights for the works on this site are retained by the individual authors and/or other copyright owners. Terms and conditions for use of this material are defined in the policies page. 
Table 2. Study methods and participant characteristics of included studies.

\begin{tabular}{|c|c|c|c|c|c|c|c|c|}
\hline Study ID & [1] Allen, 2009 & [2] Boss, 2014 & [3] Byers, 2010 & [4] Damush, 2011 & [5] Faulkner, 2015 & [6] Faulkner, 2017 & [7] Gilham, 2010 & [8] Green, 2007 \\
\hline $\begin{array}{l}\text { Rationale } \\
\text { and aims }\end{array}$ & $\begin{array}{l}\text { To compare comprehensive } \\
\text { interdisciplinary post- } \\
\text { discharge stroke care } \\
\text { management to organised } \\
\text { stroke department } \\
\text { enhanced discharge } \\
\text { planning. }\end{array}$ & $\begin{array}{l}\text { To investigate the feasibility } \\
\text { and safety of a post-stroke } \\
\text { care with exercise program. }\end{array}$ & $\begin{array}{l}\text { To develop and evaluate an } \\
\text { enhanced method of } \\
\text { teaching stroke education in } \\
\text { an acute setting. }\end{array}$ & $\begin{array}{l}\text { To test the feasibility and } \\
\text { effectiveness of a stroke- } \\
\text { specific self-management } \\
\text { ntervention on functioning } \\
\text { and quality of life. }\end{array}$ & $\begin{array}{l}\text { To assess the effects of a } \\
\text { post-stroke exercise and } \\
\text { education program on short- } \\
\text { and long-term psychosocial } \\
\text { health outcomes. }\end{array}$ & $\begin{array}{l}\text { To assess the long-term } \\
\text { effects of a post-stroke } \\
\text { exercise and education } \\
\text { program on clinical outcome } \\
\text { measures and costs } \\
\text { associated with hospital } \\
\text { admissions. }\end{array}$ & $\begin{array}{l}\text { To evaluate the significance } \\
\text { of enhanced secondary } \\
\text { prevention on readiness to } \\
\text { change health behaviour } \\
\text { post-stroke compared to } \\
\text { conventional secondary } \\
\text { prevention. }\end{array}$ & $\begin{array}{l}\text { To examine the impact of } \\
\text { one-to-one nurse/patient } \\
\text { interviews on the stroke } \\
\text { knowledge and behaviour } \\
\text { change. }\end{array}$ \\
\hline $\begin{array}{l}\text { Design/ } \\
\text { Allocation }\end{array}$ & $\begin{array}{l}\text { RCT, block sequence } \\
\text { andomisation. Allocation } \\
\text { through sealed envelopes. }\end{array}$ & $\begin{array}{l}\text { RCT, allocation sequence } \\
\text { generated by coin tossing. } \\
\text { Randomisation through } \\
\text { sealed envelopes. }\end{array}$ & $\begin{array}{l}\text { RCT, randomisation } \\
\text { conducted using birth date. }\end{array}$ & $\begin{array}{l}\mathrm{RCT} \text {, block randomisation } \\
\text { stratified by site and } \\
\text { inpatient rehabilitation. }\end{array}$ & $\begin{array}{l}\text { RCT, parallel group. } \\
\text { Randomised by computer } \\
\text { generator. }\end{array}$ & RCT. & $\begin{array}{l}\text { RCT, block randomisation } \\
\text { was stratified and } \\
\text { completed using computer } \\
\text { generated codes. }\end{array}$ & $\begin{array}{l}\text { RCT, allocation through } \\
\text { sealed envelopes. }\end{array}$ \\
\hline Blinding & Not reported. & Single-blinded. & Not reported. & $\begin{array}{l}\text { Assessors were blind to } \\
\text { reatment assignment. }\end{array}$ & $\begin{array}{l}\text { Participants and programme } \\
\text { practitioner were aware of } \\
\text { allocation. Outcome } \\
\text { assessors were blind to } \\
\text { allocation. }\end{array}$ & Not reported. & Single-blinded. & $\begin{array}{l}\text { Participants and study } \\
\text { coordinator were aware of } \\
\text { allocation. }\end{array}$ \\
\hline Duration & 6 months & 12 months & 1 month & 6 months & 12 months & 3.5 years & 3 months & 3 months \\
\hline Setting & Home-based. USA. & $\begin{array}{l}\text { Specialised stroke unit. } \\
\text { Netherlands. }\end{array}$ & $\begin{array}{l}\text { Suburban tertiary care } \\
\text { hospital. USA. }\end{array}$ & $\begin{array}{l}\text { Two Veteran hospitals. } \\
\text { USA. }\end{array}$ & $\begin{array}{l}\text { Hospital and academic } \\
\text { institution. New Zealand. }\end{array}$ & $\begin{array}{l}\text { Hospital records. New } \\
\text { Zealand }\end{array}$ & $\begin{array}{l}\text { Outpatient clinic at district } \\
\text { hospital. UK. }\end{array}$ & $\begin{array}{l}\text { Ambulatory stroke } \\
\text { prevention clinic. Canada. }\end{array}$ \\
\hline $\begin{array}{l}\text { Participant } \\
\text { numbers \& } \\
\text { demographics }\end{array}$ & $\begin{array}{l}\text { Diagnosis: Minor stroke, } \\
\text { NIHSS } \geq 1 \\
n=380 \text { (190 in each group) } \\
\text { Age: Intervention group } \\
\text { mean }=68 \text { years; Control } \\
\text { group mean = 69 years } \\
\text { Gender: Intervention group } \\
=48 \text { men; Control group = } \\
52 \text { men } \\
\text { Type of stroke: Ischaemic } \\
(n=380) \\
\text { Stroke severity: } \\
\text { Intervention NIISSS mean = } \\
2.0 ; \text { Control NIHSS mean = } \\
1.7\end{array}$ & $\begin{array}{l}\text { Diagnosis: TIA/Minor } \\
\text { stroke, NIHSS } \leq 3 \\
n=20(10 \text { in each group) } \\
\text { Age: Intervention group } \\
\text { mean }=62.4 \text { years; Control } \\
\text { group mean }=63 \text { years } \\
\text { Gender: Intervention group } \\
=7 \text { men; Control group }=7 \\
\text { men } \\
\text { Type of stroke: TIA } \\
\text { (Intervention } n=5 \text {, Control } \\
=3 \text { ); Minor stroke } \\
(\text { Intervention } n=5 \text {, Control } \\
n=7)\end{array}$ & $\begin{array}{l}\text { Diagnosis: TIA/lschaemic } \\
\text { stroke, NIHSS } \leq 15 \\
n=20 \text { randomised } \\
\text { Age: Intervention group } \\
\text { mean = } 58.2 \text { years; Control } \\
\text { group mean = 58.1 years } \\
\text { Gender: Intervention group } \\
=3 \text { men, } 2 \text { women; Control } \\
\text { group = } 4 \text { men, } 4 \text { women. } \\
\text { Stroke severity: } \\
\text { Intervention NIHSS mean = } \\
2.0 ; \text { Control NIHSS mean = } \\
1.0\end{array}$ & $\begin{array}{l}\text { Diagnosis: Stroke } \\
n=63 \text { (Intervention } n=30 ; \\
\text { Control } n=33 \text { ) } \\
\text { Age: Intervention group } \\
\text { mean = 67.3 years; } \\
\text { Control group mean = 64 } \\
\text { years } \\
\text { Gender: Intervention group } \\
=30 \text { men; Control group = } \\
32 \text { men, } 1 \text { woman } \\
\text { Stroke severity: } \\
\text { Intervention NIHSS mean = } \\
\text { 3.27; Control NIHSS mean } \\
=3.33\end{array}$ & $\begin{array}{l}\text { Diagnosis: TIA/Mild stroke, } \\
\text { within } 7 \text { days of onset } \\
n=60(30 \text { in each group) } \\
\text { Age: Intervention group } \\
\text { mean }=65 \text { ( } \pm 11 \text { ) years; } \\
\text { Control group mean }= \\
68( \pm 10 \text { ) years } \\
\text { Gender: Intervention group } \\
=15 \text { men, } 12 \text { women; } \\
\text { Control group }=14 \text { men, } 14 \\
\text { women } \\
\text { Type of stroke: TIA } \\
\text { (Intervention } n=16 \text {, Control } \\
n=17) ; \text { Mild stroke } \\
\text { (Intervention } n=11 \text {, Control } \\
n=11)\end{array}$ & $\begin{array}{l}\text { Diagnosis: TIA/Minor } \\
\text { stroke, within } 2 \text { weeks of } \\
\text { onset } \\
n=60 \text { ( } 30 \text { in each group) } \\
\text { Age: Intervention group } \\
\text { mean }=68 \text { ( } \pm 11 \text { ) years; } \\
\text { Control group mean } \\
69( \pm 10) \text { years } \\
\text { Gender: Intervention group } \\
=16 \text { men, } 14 \text { women; } \\
\text { Control group }=15 \text { men, } 15 \\
\text { women } \\
\text { Type of stroke: TIA } \\
\text { (Intervention } n=2 \text {, Control } \\
h=6) ; \text { Minor stroke } \\
\text { (Intervention } n=1 \text {, Control } \\
h=5) \\
\text { History: No previous } \\
\text { TIA/stroke }\end{array}$ & $\begin{array}{l}\text { Diagnosis: TIA/Minor } \\
\text { stroke } \\
\text { n }=52 \text { (26 in each group) } \\
\text { Age: Intervention group } \\
\text { mean }=67.7 \text { years; Control } \\
\text { group mean }=68.9 \text { years } \\
\text { Gender: } 15 \text { women, } 37 \\
\text { men } \\
\text { History: No previous } \\
\text { TIA/stroke }\end{array}$ & $\begin{array}{l}\text { Diagnosis: TIA/Minor } \\
\text { stroke, MMSE score }>24 \\
\mathrm{n}=200 \text { (100 in each group) } \\
\text { Age: Intervention group } \\
\text { mean = 66.26 years; } \\
\text { Control group mean = 67.24 } \\
\text { years } \\
\text { Gender: Intervention group } \\
=41 \text { women; Control group } \\
=41 \text { women } \\
\text { Previous TIA/stroke: } \\
\text { Intervention group } \mathrm{n}=26 ; \\
\text { Control group } \mathrm{n}=30\end{array}$ \\
\hline $\begin{array}{l}\text { Inclusion } \\
\text { criteria }\end{array}$ & $\begin{array}{l}\text { 1. Diagnosis of ischemic } \\
\text { stroke. } \\
\text { 2. NIHSS score } \geq 1 \\
\text { 3. Discharged to home from } \\
\text { the acute care hospital or } \\
\text { discharged to home } \\
\text { within } 8 \text { weeks from a } \\
\text { short-term nursing or } \\
\text { rehabilitation facility } \\
\text { 4. Live within } 25 \text { miles } \\
\text { 5. Have no other illness that } \\
\text { would affect post-stroke } \\
\text { care } \\
\text { 6. Speak English. } \\
\text { 7. Do not have an } \\
\text { endarterectomy planned }\end{array}$ & $\begin{array}{l}\text { 1. Aged } 18 \text { years or over. } \\
\text { 2. NIHSS score } \leq 3 \text {. } \\
\text { 3. Experienced the onset of } \\
\text { symptoms less than } 1 \\
\text { month ago } \\
\text { 4. Can walk independently } \\
\text { 5. Discharged without need } \\
\text { for further rehabilitation }\end{array}$ & $\begin{array}{l}\text { 1. Diagnosis of ischaemic } \\
\text { stroke/TIA } \\
\text { 2. Aged between } 18-90 \\
\text { years old } \\
\text { 3. NIHSS score } \leq 15 \\
\text { 4. MMSE score } \geq 20 \\
\text { 5. Have a family } \\
\text { member/significant other } \\
\text { able to participate }\end{array}$ & $\begin{array}{l}\text { 1. Diagnosis of ischaemic } \\
\text { stroke within the previous } \\
\text { month } \\
\text { 2. Aged } 18 \text { years or over } \\
\text { 3. Able to communicate and } \\
\text { understand English } \\
\text { 4. No severe cognitive } \\
\text { impairments } \\
\text { 5. Willing to attend follow- } \\
\text { ups } \\
\text { 6. Access to telephone } \\
\text { 7. Have a life expectancy of } \\
\text { at least } 12 \text { months }\end{array}$ & $\begin{array}{l}\text { 1. Diagnosis of TIA/mild } \\
\text { stroke within } 7 \text { days of } \\
\text { onset }\end{array}$ & $\begin{array}{l}\text { 1. Diagnosed with first TIA } \\
\text { or minor stroke } \\
\text { 2. Lived within the local } \\
\text { district health board } \\
\text { catchment }\end{array}$ & $\begin{array}{l}\text { 1. Diagnosed with first } \\
\mathrm{TIA} / \text { minor stroke }\end{array}$ & $\begin{array}{l}\text { 1. MMSE score }>24 \text {. } \\
\text { 2. Able to speak and read } \\
\text { English } \\
\text { 3. Able to complete } \\
\text { questionnaires in-person } \\
\text { and over the phone } \\
\text { 4. Able to attend a lifestyle } \\
\text { class } \\
\text { 5. Can provide informed } \\
\text { consent }\end{array}$ \\
\hline
\end{tabular}




\begin{tabular}{|c|c|c|c|c|c|c|c|c|}
\hline $\begin{array}{l}\text { Exclusion } \\
\text { criteria }\end{array}$ & Not detailed. & $\begin{array}{l}\text { 1. Diagnosis of dementia } \\
\text { 2. MMSE score <24 } \\
\text { 3. Aphasia, or unable to } \\
\text { speak Dutch } \\
\text { 4. Cardiopulmonary } \\
\text { contraindication for } \\
\text { physical exercise and } \\
\text { exercise testing } \\
\text { 5. Have a chronic disease } \\
\text { with an expected survival } \\
\text { of less than } 2 \text { years }\end{array}$ & $\begin{array}{l}\text { 1. Diagnosis } \\
\text { of haemorrhagic stroke } \\
\text { or coma } \\
\text { 2. NIHSS }>15 \\
\text { 3. Unable to speak English } \\
\text { 4. Unbale to commit to a 1- } \\
\text { month follow-up }\end{array}$ & $\begin{array}{l}\text { 1. Significant language } \\
\text { difficulties } \\
\text { 2. Severe dementia or other } \\
\text { cognitive impairments }\end{array}$ & $\begin{array}{l}\text { 1. Have an unstable cardiac } \\
\text { condition } \\
\text { 2. Have uncontrolled } \\
\text { diabetes } \\
\text { 3. Have severe claudication } \\
\text { 4. Oxygen dependence } \\
\text { 5. Severe dementia } \\
\text { 6. Unable to participate in } \\
\text { exercise } \\
\text { 7. Unable to speak English }\end{array}$ & $\begin{array}{l}\text { 1. Have an unstable cardiac } \\
\text { condition } \\
\text { 2. Have uncontrolled } \\
\text { diabetes } \\
\text { 3. Have severe claudication } \\
\text { 4. Oxygen dependence } \\
\text { 5. Severe dementia } \\
\text { 6. Unable to participate in } \\
\text { exercise } \\
\text { 7. Unable to speak English }\end{array}$ & Not detailed. & $\begin{array}{l}\text { 1. Unable to understand } \\
\text { English } \\
\text { 2. Have significant cognitive } \\
\text { impairment } \\
\text { 3. Unable to attend a } \\
\text { lifestyle class } \\
\text { 4. Unable to provide } \\
\text { informed consent }\end{array}$ \\
\hline Control group & $\begin{array}{l}\text { Usual post-discharge care } \\
\text { from their primary care } \\
\text { physician. } \\
\text { Both groups received a } \\
\text { discharge pack, including } \\
\text { discharge plans and stroke } \\
\text { risk factor lists, from their } \\
\text { hospital. }\end{array}$ & $\begin{array}{l}\text { Usual post-TIA/minor stroke } \\
\text { care, which involved two-to- } \\
\text { three outpatient clinic visits. }\end{array}$ & $\begin{array}{l}\text { Standard post-stroke } \\
\text { information (verbal and } \\
\text { written) at discharge. }\end{array}$ & $\begin{array}{l}\text { Written stroke education } \\
\text { materials. } \\
\text { Leaflets with schedule of } \\
\text { elephone calls for follow- } \\
\text { ups. }\end{array}$ & $\begin{array}{l}\text { Standard secondary } \\
\text { prevention and educational } \\
\text { information materials } \\
\text { provided at discharge. }\end{array}$ & $\begin{array}{l}\text { Usual pharmacological } \\
\text { management care. }\end{array}$ & $\begin{array}{l}\text { No additional secondary } \\
\text { prevention advice. }\end{array}$ & $\begin{array}{l}\text { Standard stroke prevention } \\
\text { discussion in clinic and } \\
\text { widely available education } \\
\text { materials. }\end{array}$ \\
\hline Dropouts & Dropouts $n=0$ & $\begin{array}{l}\text { Dropouts } \mathbf{n}=\mathbf{2} \\
\text { Lack of motivation } n=1 \\
\text { Cardiac complaints } n=1\end{array}$ & $\begin{array}{l}\text { Dropouts } \mathbf{n}=7 \\
\text { Hospital admission } n=2 \\
\text { Lost to contact } n=5\end{array}$ & $\begin{array}{l}\text { Dropouts } n=3 \\
\text { Lost to contact } n=1 \\
\text { Withdrew consent } n=1 \\
\text { Discharged to other service } \\
n=1\end{array}$ & $\begin{array}{l}\text { Dropouts } \mathbf{n}=\mathbf{5} \\
\text { Diagnosis of depression } \\
n=2 \\
\text { Migration } n=1 \\
\text { Lack of time } n=1 \\
\text { Death } n=1\end{array}$ & $\begin{array}{l}\text { Dropouts } \mathbf{n}=9 \\
\text { Death } n=1 \\
\text { Diagnosis of depression } n= \\
2 \\
\text { Lack of time } n=4 \\
\text { Migration } n=1 \\
\text { Uncontrolled atrial fibrillation } \\
n=1\end{array}$ & $\begin{array}{l}\text { Dropouts } \mathbf{n = 2} \\
\text { Hospital admission } n=1 \\
\text { Hearing difficulties } n=1\end{array}$ & $\begin{array}{l}\text { Dropouts } n=36 \\
\text { Discontinued intervention } n \\
=21 \\
\text { Withdrew consent } n=3 \\
\text { Withdrew due to illness } n=4 \\
\text { Death } n=1 \\
\text { Did not meet study criteria } n \\
=4 \\
\text { Randomised in illness } n=3\end{array}$ \\
\hline
\end{tabular}


Table 2. Continued.

\begin{tabular}{|c|c|c|c|c|c|c|c|}
\hline Study ID & [9] Herron, 2017 & [10] Heron, 2019 & [11] Rochette, 2013 & [12] Sajatovic, 2018 & [13] Wang, 2013 & [14] Wolf, 2016 & [15] Wolf, 2017 \\
\hline $\begin{array}{l}\text { Rationale } \\
\text { and aims }\end{array}$ & $\begin{array}{l}\text { To evaluate the feasibility of } \\
\text { conducting a trial of an adapted } \\
\text { home-based cardiac } \\
\text { rehabilitation programme, 'The } \\
\text { Healthy Brain Rehabilitation } \\
\text { Manual', for patients following a } \\
\text { TIA/minor stroke, participants' } \\
\text { views on the intervention and, to } \\
\text { identify the behaviour change } \\
\text { techniques (BCTs) used. }\end{array}$ & $\begin{array}{l}\text { To pilot a randomised controlled } \\
\text { trial of a novel home-based } \\
\text { prevention programme ('The } \\
\text { Healthy Brain Rehabilitation } \\
\text { Manual') for patients with TIA or } \\
\text { 'minor' stroke. }\end{array}$ & $\begin{array}{l}\text { To determine whether a low- } \\
\text { cost, multimodal support } \\
\text { intervention (WE CALL) offered } \\
\text { for } 6 \text { months would be effective } \\
\text { compared to the availability of a } \\
\text { resource person (YOU CALL) } \\
\text { for } 6 \text { months, in TIA/minor } \\
\text { stroke patients. }\end{array}$ & $\begin{array}{l}\text { To evaluate the effectiveness of } \\
\text { a novel Behavioral Targeted } \\
\text { Management Intervention } \\
\text { (TEAM) vs. usual care in } \\
\text { African American (AA) men with } \\
\text { TIA or minor stroke. }\end{array}$ & $\begin{array}{l}\text { To evaluate whether patients } \\
\text { with mild stroke who received a } \\
\text { community-based stroke } \\
\text { nursing education and } \\
\text { rehabilitation programme had } \\
\text { better knowledge, behaviour } \\
\text { and self-efficacy compared with } \\
\text { those who were exposed to } \\
\text { traditional education } \\
\text { programmes in hospital setting. }\end{array}$ & $\begin{array}{l}\text { To evaluate the Improving } \\
\text { Participation after Stroke Self- } \\
\text { Management Program (IPASS) } \\
\text { in improving self-efficacy and } \\
\text { participation in everyday life } \\
\text { activities for individuals living } \\
\text { with the long-term } \\
\text { consequences of stroke. }\end{array}$ & $\begin{array}{l}\text { To evaluate the feasibility and } \\
\text { effect of the Chronic Disease } \\
\text { Self-Management Program } \\
\text { (CDSMP) for people after mild } \\
\text { stroke. }\end{array}$ \\
\hline \begin{tabular}{|l|} 
Design// \\
Allocation
\end{tabular} & $\begin{array}{l}\text { RCT. Computer generated } \\
\text { randomisation. }\end{array}$ & $\begin{array}{l}\text { RCT. Random allocation } \\
\text { through sealed envelopes. }\end{array}$ & RCT. Random allocation. & $\begin{array}{l}\text { RCT. Computer generated } \\
\text { randomisation. }\end{array}$ & RCT. Random cluster sampling & RCT. Block randomisation. & $\begin{array}{l}\text { RCT. Random number } \\
\text { generator. }\end{array}$ \\
\hline Blinding & Not blinded. & $\begin{array}{l}\text { Single-blinded trial. Research } \\
\text { nurse, blinded to intervention } \\
\text { allocation, undertook post- } \\
\text { intervention assessments. }\end{array}$ & Single-blinded trial. & Not blinded. & Not blinded. & $\begin{array}{l}\text { Single-blinded trial. Assessor } \\
\text { blinded. }\end{array}$ & $\begin{array}{l}\text { Single-blinded trial. Assessor } \\
\text { plinded. }\end{array}$ \\
\hline Duration & 6 weeks & 12 weeks & 12 months & 6 months & 6 months & 9 months & 6 months \\
\hline Setting & $\begin{array}{l}\text { Patients attending TIA/ 'minor' } \\
\text { stroke assessment hospital } \\
\text { clinics in Belfast }\end{array}$ & $\begin{array}{l}\text { 'Drop-in' TIA clinics and } \\
\text { outpatient clinics in four } \\
\text { TIAI/minor' stroke assessment } \\
\text { units in different Northern } \\
\text { Ireland Health and Social Care } \\
\text { Trusts. }\end{array}$ & $\begin{array}{l}11 \text { acute care hospitals located } \\
\text { in urban and rural areas across } \\
4 \text { Canadian provinces. }\end{array}$ & $\begin{array}{l}\text { The clinical practices of study } \\
\text { nvestigators at } 2 \text { large } \\
\text { academic medical centres. } \\
\text { USA }\end{array}$ & $\begin{array}{l}\text { Outpatients of the neurology } \\
\text { department of Chung Shan } \\
\text { Medical University Hospital. } \\
\text { Seven municipal communities. } \\
\text { Taiwan }\end{array}$ & $\begin{array}{l}\text { Two University settings both } \\
\text { with relationships to free- } \\
\text { standing rehabilitation } \\
\text { hospitals. } \\
\text { USA }\end{array}$ & $\begin{array}{l}\text { Home based. } \\
\text { University-affiliated acute care } \\
\text { hospital. } \\
\text { USA }\end{array}$ \\
\hline \begin{tabular}{|l} 
Participant \\
numbers \& \\
demographics
\end{tabular} & 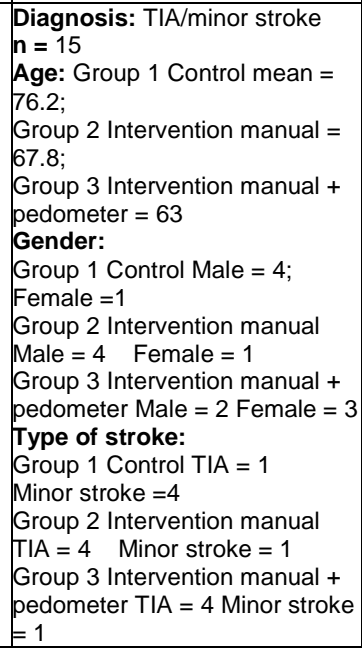 & $\begin{array}{l}\text { Diagnosis: TIA/Minor stroke, } \\
\text { within } 4 \text { weeks of onset } \\
n=40 \text { (GP Intervention } n=14 ; \\
\text { Stroke nurse Intervention } n= \\
14 ; \text { Control } n=12) \\
\text { Age: } \\
\text { GP Intervention group mean = } \\
65.71 \text { years; Stroke nurse } \\
\text { Intervention group mean = } \\
63.29 \text { years; Control group } \\
\text { mean }=69.67 \text { years } \\
\text { Gender: GP Intervention group } \\
=9 \text { men, } 5 \text { women; Stroke } \\
\text { nurse Intervention group }=7 \\
\text { men, } 7 \text { women; Control group = } \\
8 \text { men, } 4 \text { women } \\
\text { Type of stroke: } \\
\text { TIA } n=27(n=6 \text { control) } \\
\text { Minor stroke } n=13(n=6 \\
\text { control) }\end{array}$ & $\begin{array}{l}\text { Diagnosis: Mild } \\
\text { stroke defined as a } \\
\text { score }>8.5 / 11.5 \text { on the } \\
\text { Canadian Neurological Scale or } \\
\text { a modified Rankin Score } \\
\text { between } 0 \text { and } 2 . \\
\text { n }=186 \\
\text { Age (mean): } \\
\text { Total sample }=62.5 \\
\text { Control }=63.2 \\
\text { Intervention }=61.7 \\
\text { Gender: } \\
\text { Total= } 79 \text { Female } \\
\text { Control= } 44 \text { Female } \\
\text { Intervention }=35 \text { Female } \\
\text { Type of stroke: } \\
\text { Ischaemic }=172(92.5 \%) \\
\text { Haemorrhagic }=10(5.4 \%) \\
\text { Other }=4(2.1 \%)\end{array}$ & $\begin{array}{l}\text { Diagnosis: AA men with TIA } \\
\text { within the past } 5 \text { years or minor } \\
\text { stroke defined as Barthel Index } \\
\text { score of }>60 \\
n=38 \text { (Intervention } n=19 \\
\text { Control } n=19) \\
\text { Age: Mean }=52.1 \\
\text { Gender: } 38 \text { male }(100 \%) \\
\text { Type of stroke: } \text { TIA } n=19 \\
\text { Ischaemic stroke } n=19\end{array}$ & $\begin{array}{l}\text { Diagnosis: Minor } \\
\text { stroke confirmed by positive } \\
\text { findings on CT or MRI of the } \\
\text { head, score greater than or } \\
\text { equal to } 65 \text { on the Barthel } \\
\text { Index } \\
n=\text { total }=170, \\
\text { Intervention } n=65 \\
\text { Age: } \\
\text { Control: } 67.2(\mathrm{SD}=10.4) \\
\text { Intervention: } 67.3 \\
\text { Gender: } \\
\text { Control: } \mathrm{n}=40 \text { male, } \mathrm{n}=22 \\
\text { female } \\
\text { Intervention: } \mathrm{n}=43 \text { male, } \mathrm{n}=22 \\
\text { female } \\
\text { Type of stroke: } \\
\text { ischaemic } \mathrm{n}=50 \\
\text { Haemorrhagic } \mathrm{n}=15\end{array}$ & $\begin{array}{l}\text { Diagnosis: mild or moderate } \\
\text { stroke (i.e., NIH stroke score } \leq \\
16) \\
n=185 \\
\text { Age: Control: } 59(45-80) \\
\text { Intervention: } 57(32-93) \\
\text { Gender: Control: male } \mathrm{n}=15, \\
\text { female } n=16 \\
\text { Intervention: male } \mathrm{n}=31 \text {, female } \\
n=35 \\
\text { Stroke severity: } \\
\text { NIHSS (mean) } \\
\text { Control: } 4.8(1-12) \\
\text { Intervention: } 4.7(1-12)\end{array}$ & $\begin{array}{l}\text { Diagnosis: Minor } \\
\text { stroke Definition: a mild stroke } \\
\text { as NIHSS total scores } 0-5 \\
\mathrm{n}=71 \\
\text { Age (mean): } \\
\text { Control }=62.65 \\
\text { Intervention }=57.67 \\
\text { Gender: } \\
\text { Control: female } n=14 \text {, male } \\
n=13 \\
\text { Intervention: female } n=9 \text {, male } \\
n=9\end{array}$ \\
\hline $\begin{array}{l}\text { Inclusion } \\
\text { criteria }\end{array}$ & $\begin{array}{l}\text { 1. Aged } 18 \text { years or older } \\
\text { 2. Within } 4 \text { weeks of their first } \\
\text { symptoms of a TIA or 'mild' } \\
\text { stroke. } \\
\text { 3. Using the TOAST } \\
\text { classification system only } \\
\text { TIAs and 'minor' strokes } \\
\text { attributed to atherosclerosis } \\
\text { or small vessel occlusion } \\
\text { were included }\end{array}$ & $\begin{array}{l}\text { 1. Aged } 18 \text { years or older } \\
\text { 2. Within } 4 \text { weeks of their first } \\
\text { TIA or 'mild' stroke } \\
\text { symptoms, with diagnoses } \\
\text { attributed to atherosclerosis } \\
\text { or small vessel occlusion }\end{array}$ & $\begin{array}{l}\text { 1. Adults who sustained a first } \\
\text { mild stroke defined as a } \\
\text { score }>8.5 / 11.5 \text { on the } \\
\text { Canadian Neurological Scale } \\
\text { or a modified Rankin Score } \\
\text { between } 0 \text { and } 2 \text { on } \\
\text { admission } \\
\text { 2. Discharged home within } 3 \\
\text { weeks of the index event } \\
\text { 3. Telephone access } \\
\text { 4. Ability to understand basic } \\
\text { instructions and express } \\
\text { basic needs. } \\
\text { 5. Ability to communicate in } \\
\text { English or French }\end{array}$ & $\begin{array}{l}\text { 1. Self-identified African } \\
\text { American (AA) male } \\
\text { 2. Aged over } 65 \text { years } \\
\text { 3. Planned or recent home } \\
\text { discharge } \\
\text { 4. Barthel Index (BI) score of } \\
>60\end{array}$ & $\begin{array}{l}\text { 1. Score } \geq 20 \text { on the Mini } \\
\text { Mental Sate Examination } \\
\text { 2. No history of psychiatric } \\
\text { illness } \\
\text { 3. Score } \geq 65 \text { on the Barthel } \\
\text { Index, which references } \\
\text { performance of the basic } \\
\text { activities of daily living }\end{array}$ & $\begin{array}{l}\text { 1. Aged } 18 \text { years or older } \\
\text { 2. Mild or moderate stroke (i.e., } \\
\text { NIHSS } \leq 16 \text { ) } \\
\text { 3. At least } 3 \text { months post-stroke } \\
\text { 4. Reside in a community-based } \\
\text { setting } \\
\text { 5. Completed initial } \\
\text { acute/rehabilitation care }\end{array}$ & $\begin{array}{l}\text { 1. Mild stroke as NIHSS total } \\
\text { scores 0-5 } \\
\text { 2. English speaking } \\
\text { 3. Individuals greater than } 18 \\
\text { years of age; and } \\
\text { 4. Identified as having at least } \\
\text { one chronic condition (Work } \\
\text { Ability Index survey) }\end{array}$ \\
\hline
\end{tabular}




\begin{tabular}{|c|c|c|c|c|c|c|c|}
\hline \begin{tabular}{|l}
$\begin{array}{l}\text { Exclusion } \\
\text { criteria }\end{array}$ \\
\end{tabular} & $\begin{array}{l}\text { 1. Unstable cardiac conditions } \\
\text { 2. Contra-indications for } \\
\text { exercise training by } \\
\text { screening patients using the } \\
\text { Physical Activity Readiness } \\
\text { Questionnaire (PAR-Q) } \\
\text { 3. Unable to give informed } \\
\text { consent } \\
\text { 4. Had a previous } \\
\text { cerebrovascular event }\end{array}$ & \begin{tabular}{|l|} 
1. Unstable cardiac conditions \\
2. Contraindications for exercise \\
training or a previous \\
cerebrovascular event were \\
excluded
\end{tabular} & $\begin{array}{l}\text { 1. Individuals with moderate or } \\
\text { severe cognitive deficits } \\
\text { (based on clinical judgement) } \\
\text { 2. Those who experienced } \\
\text { another stroke before } \\
\text { baseline measures were } \\
\text { completed were excluded }\end{array}$ & $\begin{array}{l}\text { 1. AA men unwilling or unable } \\
\text { to provide informed consent }\end{array}$ & $\begin{array}{l}\text { 1. Participants could not } \\
\text { have severe language and } \\
\text { hearing impairments that } \\
\text { could interfere with } \\
\text { evaluation interviews }\end{array}$ & $\begin{array}{l}\text { 1. Not medically stable } \\
\text { 2. Were moderately or severely } \\
\text { cognitively impaired (i.e., } \\
\text { short blessed cognitive test } \\
\text { score }>8 \text { ) } \\
\text { 3. Had severe aphasia (i.e., } \\
\text { Boston diagnostic aphasia } \\
\text { exam score }<9 ; 15 \text { item } \\
\text { Boston naming test }<10 \text { ) }\end{array}$ & $\begin{array}{l}\text { 1. Severe aphasia (NIHSS } \\
\text { aphasia score=2) } \\
\text { 2. Moderate to severe cognitive } \\
\text { impairment (Montreal } \\
\text { Cognitive Assessment } \\
\text { (MOCA) Score }<21 \text { ) } \\
\text { 3. History of dementia } \\
\text { 4. Haemorrhagic stroke } \\
\text { 5. Neurological diagnoses other } \\
\text { than stroke } \\
\text { 6. Major psychiatric illness } \\
\text { 7. Terminal illness } \\
\text { 8. Score of no-higher than } 20 \\
\text { on the PHQ-9 indicating } \\
\text { significant depressive } \\
\text { symptoms } \\
\text { 9. Aged over } 90 \text { years }\end{array}$ \\
\hline Control group & $\begin{array}{l}\text { Current standard post-TIA/minor } \\
\text { stroke care as per current UK } \\
\text { guidelines. }\end{array}$ & $\begin{array}{l}\text { Standard post-TIA/minor stroke } \\
\text { care. } \\
\text { Follow-up calls from a GP. }\end{array}$ & $\begin{array}{l}\text { Standard of care - contact with } \\
\text { medical staff initiated by } \\
\text { patients themselves (YOU } \\
\text { CALL). }\end{array}$ & $\begin{array}{l}\text { Treatment with regular medical } \\
\text { care providers. Beyond follow- } \\
\text { up research assessments at the } \\
\text { same time points as TEAM, } \\
\text { there was no interaction } \\
\text { between the participants and } \\
\text { the research team. }\end{array}$ & Normal care group. & $\begin{array}{l}\text { Control group started a 12-week } \\
\text { waiting period and did not } \\
\text { receive any active research } \\
\text { intervention at this time. } \\
\text { Participants completed another } \\
\text { baseline assessment following } \\
\text { this } 12 \text {-week wait, prior to being } \\
\text { scheduled to complete the } \\
\text { IPASS. }\end{array}$ & $\begin{array}{l}\text { Control group did not receive } \\
\text { any active intervention as a part } \\
\text { of this study, but they were } \\
\text { offered the opportunity to } \\
\text { complete the CDSMP after their } \\
\text { participation in this study had } \\
\text { ended. }\end{array}$ \\
\hline Dropouts & Dropouts $n=0$ & $\begin{array}{l}\text { Dropouts } \mathbf{n}=\mathbf{1} \text { (work } \\
\text { commitments) }\end{array}$ & $\begin{array}{l}\text { Dropouts } n=84 \\
\text { 6-month follow up }-n=37 \\
\text { unreachable } \\
\text { 12-month follow up - } n=47 \\
\text { unreachable }\end{array}$ & $\begin{array}{l}\text { There were } 28 \text { individuals ( } 14 \text { in } \\
\text { TEAM and } 14 \text { in TAU) able to } \\
\text { be assessed at } 12 \text {-weeks and } \\
28 \text { individuals ( } 14 \text { in TEAM and } \\
14 \text { in TAU) able to be assessed } \\
\text { at } 24 \text {-weeks. Drop-out at } 24- \\
\text { weeks was } 26.3 \% \text {. }\end{array}$ & $\begin{array}{l}\text { Dropouts } \mathbf{n}=\mathbf{4 3} \\
n=20 \text { in intervention group } \\
n=23 \text { in control group }\end{array}$ & $\begin{array}{l}\text { Dropouts } \mathbf{n}=\mathbf{1 9} \\
\text { Seven from the immediate } \\
\text { intervention group and } 12 \text { from } \\
\text { the wait list group were lost } \\
\text { during the 6- to 9-month period. }\end{array}$ & $\begin{array}{l}\text { Dropouts } \mathbf{n}=\mathbf{2 2} \\
\text { Control: } \\
\text { medical withdrawal } n=1 \\
\text { lost to follow-up } n=8 \\
\text { Intervention: } \\
\text { voluntary withdrawal } n=10 \\
\text { lost to } 6 \mathrm{~m} \text { follow-up } n=3\end{array}$ \\
\hline
\end{tabular}


Table 3. Intervention details of included studies.

\begin{tabular}{|c|c|c|c|c|c|c|c|c|}
\hline Study ID & [1] Allen, 2009 & [2] Boss, 2014 & [3] Byers, 2010 & [4] Damush, 2011 & [5] Faulkner, 2015 & [6] Faulkner, 2017 & [7] Gilham, 2010 & [8] Green, 2007 \\
\hline Brief name & $\begin{array}{l}\text { Post-stroke care } \\
\text { management model }\end{array}$ & MotiveS and MovelT & $\begin{array}{l}\text { Enhanced education } \\
\text { group }\end{array}$ & $\begin{array}{l}\text { Stroke self-management } \\
\text { programme }\end{array}$ & $\begin{array}{l}\text { Early exercise } \\
\text { engagement with } \\
\text { education }\end{array}$ & $\begin{array}{l}\text { Long-term effects of } \\
\text { exercise and education } \\
\text { programme }\end{array}$ & $\begin{array}{l}\text { Enhanced secondary } \\
\text { prevention }\end{array}$ & $\begin{array}{l}\text { Education-Counselling } \\
\text { Interview }(\mathrm{ECI})\end{array}$ \\
\hline $\begin{array}{l}\text { Components of } \\
\text { intervention }\end{array}$ & $\begin{array}{l}\text { - Home visits from a } \\
\text { physical therapist } \\
\text { when required. } \\
\text { - Aids to help with } \\
\text { medication adherence. } \\
\text { - Social service plans to } \\
\text { help maximise quality } \\
\text { of life. } \\
\text { - Monitoring for common } \\
\text { post-stroke } \\
\text { complications. } \\
\text { - Personalised health } \\
\text { record. } \\
\text { - Education about } \\
\text { lifestyle modification } \\
\text { and stroke warning } \\
\text { signs. }\end{array}$ & $\begin{array}{l}\text { - } 3 \text { one-hour aerobic } \\
\text { exercise and strength } \\
\text { training sessions a } \\
\text { week for } 8 \text { weeks. } \\
\text { - Participants were then } \\
\text { seen every } 3 \text { months } \\
\text { at an outpatient clinic } \\
\text { where the view was } \\
\text { maintaining an active } \\
\text { lifestyle. }\end{array}$ & $\begin{array}{l}\text { - Enhanced stroke } \\
\text { education delivered } \\
\text { through motivation } \\
\text { interviewing } \\
\text { - Individualised verbal } \\
\text { and written information } \\
\text { which included a sheet } \\
\text { detailing diagnosis, its } \\
\text { impact on recovery, } \\
\text { current medication, } \\
\text { and what to expect } \\
\text { upon discharge. } \\
\text { - Collaborative } \\
\text { approach between } \\
\text { patient, caregiver and } \\
\text { healthcare provider. }\end{array}$ & $\begin{array}{l}\text { - Telephone-based } \\
\text { stroke management } \\
\text { programme. } \\
\text { - Each session covered } \\
\text { self-management } \\
\text { topics including } \\
\text { recovering from stroke, } \\
\text { adapting to stroke- } \\
\text { related disability, and } \\
\text { understanding the } \\
\text { warning signs of future } \\
\text { stroke. } \\
\text { - Participants were } \\
\text { coached to choose } \\
\text { one or more goals per } \\
\text { session to target. } \\
\text { - Follow-up telephone } \\
\text { calls provided } \\
\text { individualised } \\
\text { feedback about goal } \\
\text { progress. }\end{array}$ & $\begin{array}{l}\text { - Twice-weekly exercise } \\
\text { and education } \\
\text { programme, delivered } \\
\text { across } 8 \text { weeks. } \\
\text { - Two } 90 \text {-minute } \\
\text { exercise sessions and } \\
\text { one } 30 \text {-min education } \\
\text { session. } \\
\text { - Each exercise session } \\
\text { contained } 30 \text { minutes } \\
\text { of aerobic exercise } \\
\text { and } 60 \text { minutes } \\
\text { resistance training. } \\
\text { - Education sessions } \\
\text { focused on stroke } \\
\text { prevention, } \\
\text { emotional/behavioural } \\
\text { changes post-TIA, and } \\
\text { risk factors. }\end{array}$ & $\begin{array}{l}\text { - 8-week exercise and } \\
\text { education programme. } \\
\text { - Employed within 2- } \\
\text { weeks after TIA/minor } \\
\text { stroke. }\end{array}$ & $\begin{array}{l}\text { - Enhanced secondary } \\
\text { prevention support. } \\
\text { - Information about } \\
\text { stroke pathology and } \\
\text { risk factors. } \\
\text { - Discussion about } \\
\text { behaviour change } \\
\text { intentions } \\
\text { - If appropriate, a plan } \\
\text { to help behaviour } \\
\text { change. } \\
\text { - Telephone support } \\
\text { and follow-up to } \\
\text { discuss progress. }\end{array}$ & $\begin{array}{l}\text { - One-to-one ECI, led by } \\
\text { nurse, conducted in a } \\
\text { stroke prevention clinic } \\
\text { visit. } \\
\text { - ECl lasted 15- to 20- } \\
\text { minutes. } \\
\text { - Content was specific } \\
\text { to participants' lifestyle } \\
\text { and personal stroke } \\
\text { risk factors. } \\
\text { - Motivational } \\
\text { interviewing was } \\
\text { employed to help } \\
\text { identify health } \\
\text { behaviours which } \\
\text { needed to change and } \\
\text { set goals which would } \\
\text { help promote that } \\
\text { change. } \\
\text { - Participants were } \\
\text { invited to attend a 3- } \\
\text { hour lifestyle class. } \\
\text { - Class provided } \\
\text { education about risk } \\
\text { factors, management, } \\
\text { and the importance of } \\
\text { health living. } \\
\text { - Participants received a } \\
\text { manual with the } \\
\text { information covered. }\end{array}$ \\
\hline $\begin{array}{l}\text { Who provided } \\
\text { (and/or set up } \\
\text { device) }\end{array}$ & $\begin{array}{l}\text { APN-CN (advanced } \\
\text { practice nurse care } \\
\text { manager) }\end{array}$ & $\begin{array}{l}\text { Specialist } \\
\text { physiotherapists }\end{array}$ & $\begin{array}{l}\text { Discharge educator } \\
\text { trained by research } \\
\text { team in motivational } \\
\text { interviewing techniques. }\end{array}$ & $\begin{array}{l}\text { Nurse, physician } \\
\text { assistant and a master } \\
\text { level social scientist. } \\
\text { All had received } 18 \\
\text { hours of standardised } \\
\text { training. } \\
\end{array}$ & $\begin{array}{l}\text { Health and exercise } \\
\text { practitioners }\end{array}$ & Not reported. & Researcher & Study nurse \\
\hline $\begin{array}{l}\text { Procedures and } \\
\text { how it was } \\
\text { delivered }\end{array}$ & $\begin{array}{l}\text { - The APN-CM made } \\
\text { home visits to conduct } \\
\text { an assessment within } \\
1 \text {-week post- } \\
\text { discharge. } \\
\text { - Standard post-stroke } \\
\text { education and } \\
\text { intervention protocols } \\
\text { were implemented } \\
\text { during the visit. } \\
\text { - Results of the } \\
\text { assessment were } \\
\text { reviewed by an } \\
\text { interdisciplinary post- } \\
\text { stroke consultation } \\
\text { team. } \\
\text { - The team developed } \\
\text { patient-specific care } \\
\text { plan and } \\
\text { communicated this to }\end{array}$ & $\begin{array}{l}\text { - An 8-week exercise } \\
\text { course was delivered } \\
\text { through } 3 \text { one-hour } \\
\text { sessions per week. } \\
\text { - } 2 \text { specialised } \\
\text { physiotherapists } \\
\text { delivered the course. } \\
\text { - Content focused on } \\
\text { aerobic exercise and } \\
\text { strength training, with } \\
\text { the intensity of the } \\
\text { exercise increased as } \\
\text { the course progressed. } \\
\text { - Exercise intensity was } \\
\text { decided on the } \\
\text { maximum heart rate } \\
\text { and power achieved } \\
\text { during a maximum } \\
\text { exercise test. } \\
\text { - Following course, } \\
\text { participants visited a }\end{array}$ & $\begin{array}{l}\text { - At point of discharge, } \\
\text { participants were given } \\
\text { written and verbal } \\
\text { education by the } \\
\text { trained educator, } \\
\text { whilst their caregiver } \\
\text { was present. } \\
\text { - Motivational } \\
\text { interviewing was used } \\
\text { to initiate a } \\
\text { collaborative approach } \\
\text { between the } \\
\text { participant, caregiver } \\
\text { and healthcare } \\
\text { provider. }\end{array}$ & $\begin{array}{l}\text { - 12-week programme } \\
\text { following discharge. } \\
\text { - Programme delivered } \\
\text { through } 6 \text { biweekly } \\
\text { telephone sessions } \\
\text { with educator. } \\
\text { - Educator followed } \\
\text { written standardised } \\
\text { manual. } \\
\text { - Sessions covered } \\
\text { stroke education and } \\
\text { self-management. } \\
\text { - Educator helped } \\
\text { participants set } \\
\text { achievable goals to } \\
\text { promote self-efficacy } \\
\text { and behaviour change. } \\
\text { - Each session provided } \\
\text { individualised }\end{array}$ & $\begin{array}{l}\text { - Exercise sessions } \\
\text { were } 30 \text { minutes of } \\
\text { aerobic exercise and } \\
60 \text { minutes of } \\
\text { resistance training. } \\
\text { - Aerobic exercise was } \\
15 \text { minutes of cycling } \\
\text { and } 15 \text { minutes of } \\
\text { treadmill. } \\
\text { - Resistance training } \\
\text { involved alternate } \\
\text { bicep curls, shoulder } \\
\text { press and squats. } \\
\text { - Education sessions } \\
\text { involved didactic group } \\
\text { discussions. } \\
\text { - Sessions were } \\
\text { designed to enable } \\
\text { health and exercise }\end{array}$ & Not reported. & $\begin{array}{l}\text { - Face-to-face } \\
\text { discussion about } \\
\text { secondary prevention } \\
\text { and behaviour change } \\
\text { intentions. } \\
\text { - Discussion in style of } \\
\text { motivational } \\
\text { interviewing. } \\
\text { - Telephone support } \\
\text { provided by research } \\
\text { team and focused on } \\
\text { progress of behaviour } \\
\text { changes. }\end{array}$ & $\begin{array}{l}\text { - 15-20-minute ECI was } \\
\text { incorporated into the } \\
\text { standard nursing } \\
\text { element of stroke } \\
\text { prevention the clinic } \\
\text { visit. } \\
\text { - Lifestyle class was } \\
\text { coordinated to occur } \\
\text { within } 1-2 \text { months of } \\
\text { clinic visit. } \\
\text { - 3-hour lifestyle class } \\
\text { had } 50-75 \text { members. } \\
\text { - Class was led by } \\
\text { nurse, nutritionist and } \\
\text { social worker. } \\
\text { - Participants who } \\
\text { attended lifestyle class } \\
\text { completed measures }\end{array}$ \\
\hline
\end{tabular}




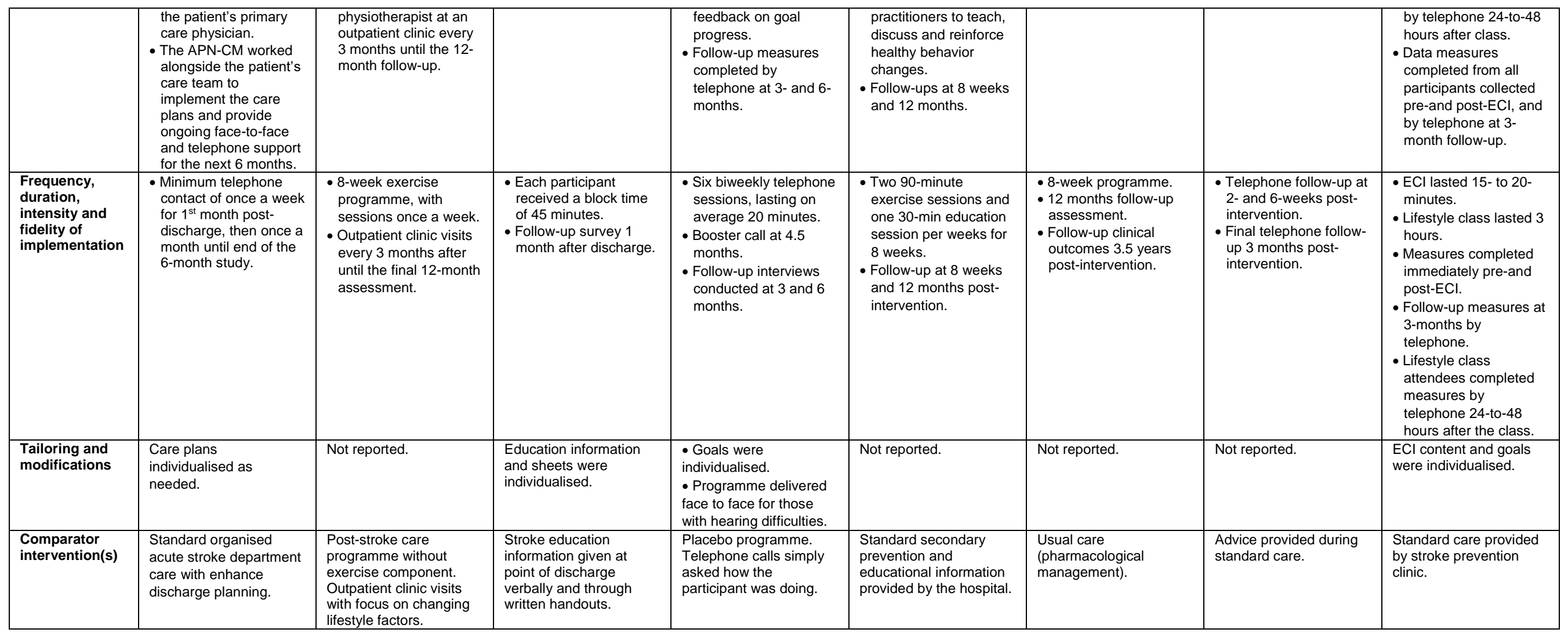


Table 3. Continued.

\begin{tabular}{|c|c|c|c|c|c|c|c|}
\hline Study ID & [9] Heron, 2017 & [10] Herron, 2019B & [11] Rochette, 2013 & [12] Sajatovic, 2018 & [13] Wang, 2013 & [14] Wolf, 2016 & [15] Wolf, 2017 \\
\hline Brief name & $\begin{array}{l}\text { The Healthy Brain } \\
\text { Rehabilitation Manual with GP } \\
\text { follow-up }\end{array}$ & $\begin{array}{l}\text { The Healthy Brain } \\
\text { Rehabilitation Manual with } \\
\text { GP or Stroke nurse follow- } \\
\text { ups }\end{array}$ & $\begin{array}{l}\text { Low-cost, multimodal } \\
\text { support intervention (WE } \\
\text { CALL). }\end{array}$ & $\begin{array}{l}\text { Behavioral Targeted } \\
\text { Management Intervention } \\
\text { (TEAM). }\end{array}$ & $\begin{array}{l}\text { A community-based stroke } \\
\text { nursing education and } \\
\text { rehabilitation programme. }\end{array}$ & $\begin{array}{l}\text { Improving Participation after } \\
\text { Stroke Self-Management } \\
\text { Program (IPASS). }\end{array}$ & $\begin{array}{l}\text { Chronic Disease Self- } \\
\text { Management Program } \\
\text { (CDSMP). }\end{array}$ \\
\hline $\begin{array}{l}\text { Components of } \\
\text { intervention }\end{array}$ & $\begin{array}{l}\text { - The Healthy Brain } \\
\text { Rehabilitation Manual } \\
\text { contained medical and social } \\
\text { information about TIAs/minor } \\
\text { strokes and how to set goals } \\
\text { and action plans for changing } \\
\text { certain aspects of their lives. } \\
\text { - Sections focusing on topics } \\
\text { relevant to cardiovascular } \\
\text { risk (smoking, physical and } \\
\text { sexual activity, mental health } \\
\text { issues (primarily anxiety and } \\
\text { depression), community } \\
\text { resources (e.g. smoking } \\
\text { cessation support; exercise } \\
\text { classes), diet and secondary } \\
\text { prevention medication). } \\
\text { - Manual was supported with } \\
\text { telephone follow-up by a } \\
\text { health professional, a GP. }\end{array}$ & $\begin{array}{l}\text { - The Healthy Brain } \\
\text { Rehabilitation Manual } \\
\text { included TIA/stroke } \\
\text { information and a stroke } \\
\text { risk reduction plan (see } \\
\text { Heron, } 2017 \text { for detail). } \\
\text { - Promoted a healthy } \\
\text { lifestyle and set pedometer } \\
\text { goals. } \\
\text { - One version of intervention } \\
\text { included standard post- } \\
\text { stroke care, the manual, } \\
\text { and a GP telephone } \\
\text { follow-up. } \\
\text { - Another version included } \\
\text { standard post-stroke care, } \\
\text { the manual and a stroke } \\
\text { nurse telephone follow-up. }\end{array}$ & $\begin{array}{l}\text { - WE CALL participants } \\
\text { received a multimodal } \\
\text { (telephone, Internet, and } \\
\text { paper) support } \\
\text { intervention. } \\
\text { - Telephone interactions } \\
\text { focused on any new or } \\
\text { ongoing issues, as well as } \\
6 \text { key areas, including } \\
\text { family functioning and } \\
\text { individualized risk factors. }\end{array}$ & $\begin{array}{l}\text { - TEAM is informed by } \\
\text { principles of social } \\
\text { cognitive theory. } \\
\text { - Key components known to } \\
\text { be critical for successful } \\
\text { post-stroke care include } \\
\text { content focused on patient } \\
\text { and care partner needs, } \\
\text { practice in problem- } \\
\text { solving, and attention to } \\
\text { emotional and role } \\
\text { management. } \\
\text { - Telephone sessions were } \\
\text { between the African } \\
\text { American (AA) stroke } \\
\text { survivor/TII patient and } \\
\text { Peer Educator or Nurse } \\
\text { Educator. } \\
\text { - Calls reinforced content } \\
\text { from the group sessions, } \\
\text { served as a behavioural } \\
\text { model, provided social } \\
\text { support, and facilitated } \\
\text { linkage with other care } \\
\text { providers. }\end{array}$ & $\begin{array}{l}\text { - Intervention and } \\
\text { counselling programme } \\
\text { comprised of two stroke } \\
\text { educational sessions, } \\
\text { communication seminars, } \\
\text { alternating with patient } \\
\text { support groups. } \\
\text { - Contents of the two- } \\
\text { session stroke education } \\
\text { consisted of lectures } \\
\text { regarding warning signs, } \\
\text { clinical manifestations, risk } \\
\text { factors of stroke, diet, } \\
\text { social activities and } \\
\text { rehabilitation. } \\
\text { - Education section } 1: \\
\text { Warning signs, Risk } \\
\text { factors, Diet } \\
\text { - Education section 2: Social } \\
\text { activities, Rehabilitation, } \\
\text { Communication section, } \\
\text { Support group section }\end{array}$ & $\begin{array}{l}\text { - The program used a } \\
\text { structured efficacy building } \\
\text { process that focused on } \\
\text { medical, emotional, role, } \\
\text { and participation } \\
\text { management to guide } \\
\text { participants to develop } \\
\text { skills related to problem- } \\
\text { solving, decision-making, } \\
\text { resource utilization, } \\
\text { client/provider/service } \\
\text { partnerships, action } \\
\text { planning, and self-tailoring } \\
\text { over time. } \\
\text { - For the seven stroke- } \\
\text { specific sessions, the } \\
\text { IPASS model was utilised } \\
\text { to guide the efficacy } \\
\text { building process. }\end{array}$ & $\begin{array}{l}\text { - The CDSMP is an } \\
\text { education program based } \\
\text { on the concept of self- } \\
\text { management. } \\
\text { - The CDSMP is focused on } \\
\text { three primary goals: } \\
\text { medical management; role } \\
\text { management; and } \\
\text { emotional management. }\end{array}$ \\
\hline $\begin{array}{l}\text { Who provided } \\
\text { (and/or set up } \\
\text { device) }\end{array}$ & $\begin{array}{l}\text { Author, who was a GP, trained } \\
\text { to deliver follow-up and provide } \\
\text { advice about the manual. }\end{array}$ & GP and stroke nurse & $\begin{array}{l}\text { Trained healthcare } \\
\text { professional (THCP) }\end{array}$ & $\begin{array}{l}\text { - Investigators trained } 2 \\
\text { Peer Dyads (AA men who } \\
\text { had a stroke/TIA and their } \\
\text { care partners) to provide } \\
\text { support and model } \\
\text { behaviors intended to } \\
\text { improve post-stroke care. } \\
\text { - Delivered alongside Stroke } \\
\text { Nurse Educator }\end{array}$ & $\begin{array}{l}\text { At each location, there were } \\
\text { different educators, who } \\
\text { were consistently trained in } \\
\text { this programme, provided } \\
\text { the same intervention for } \\
\text { each location. }\end{array}$ & $\begin{array}{l}\text { Sessions facilitated by an } \\
\text { occupational therapist(s) } \\
\text { and/or a peer facilitator with } \\
\text { stroke (depending on } \\
\text { availability) who has } \\
\text { completed the chronic } \\
\text { disease self-management } \\
\text { program (CDSMP) facilitator } \\
\text { training. }\end{array}$ & $\begin{array}{l}\text { The CDSMP was delivered } \\
\text { by two licensed occupational } \\
\text { therapists who were certified } \\
\text { facilitators. }\end{array}$ \\
\hline $\begin{array}{l}\text { Procedures and } \\
\text { how it was } \\
\text { delivered }\end{array}$ & $\begin{array}{l}\text { - During the initial } \\
\text { meeting/telephone contacts } \\
\text { trainer used motivational } \\
\text { interviewing techniques, } \\
\text { guided by the theory of } \\
\text { planned behaviour and } \\
\text { adopting the ' } 5 \mathrm{As} \text { ' approach } \\
\text { to behaviour change. } \\
\text { - Group } 1 \text {, telephoned at } 1 \text { and } \\
4 \text { weeks to answer any } \\
\text { questions regarding their } \\
\text { care or manual } \\
\text { - Group } 3 \text {, trainer encouraged } \\
\text { participants to self-set step } \\
\text { count targets. }\end{array}$ & $\begin{array}{l}\text { - Hospital or home-based } \\
\text { assessments conducted } \\
\text { by research team. } \\
\text { - Manual provided within } \\
\text { first } 4 \text { weeks of TIA/minor } \\
\text { stroke. } \\
\text { - Telephone follow-up calls } \\
\text { made by either GP or } \\
\text { stroke nurse at } 1,4 \text { and } 9 \\
\text { weeks, about any } \\
\text { concerns regarding care. } \\
\text { - Home-based follow-up } \\
\text { assessment conducted at } \\
12 \text { weeks. }\end{array}$ & $\begin{array}{l}\text { - Multimodal (telephone, } \\
\text { Internet, and paper). } \\
\text { - Additional written } \\
\text { information on stroke } \\
\text { management was provided } \\
\text { as needed (by regular } \\
\text { mail, e-mail, or Internet). } \\
\text { - Participants were referred } \\
\text { to local community } \\
\text { services as necessary and } \\
\text { directed to their family } \\
\text { doctor when they } \\
\text { experienced health } \\
\text { problems. }\end{array}$ & $\begin{array}{l}\text { - Seven brief home } \\
\text { telephone sessions } \\
\text { implemented over } 6 \\
\text { months, to coincide with } \\
\text { the } 1: 1 \text {, group sessions, } \\
\text { follow-up visits. } \\
\text { - Telephone sessions were } \\
\text { between the AA stroke } \\
\text { survivor/TIA patient and } \\
\text { Peer or Nurse Educator. } \\
\text { - Group sessions co-led by } \\
\text { the Nurse Educator and } \\
\text { Peer Dyad, using a } \\
\text { detailed curriculum with } \\
\text { semi-formal scripting. }\end{array}$ & $\begin{array}{l}\text { - Lectures } \\
\text { - Demonstrations } \\
\text { - Discussion/sharing } \\
\text { sessions }\end{array}$ & $\begin{array}{l}\text { - Patients learned three } \\
\text { different strategies (i.e. } \\
\text { change the person, } \\
\text { change the activity, and/or } \\
\text { change the environment) } \\
\text { to utilize to manage and } \\
\text { support their participation } \\
\text { in daily life. } \\
\text { - Location not mentioned } \\
\text { other than different study } \\
\text { sites. }\end{array}$ & $\begin{array}{l}\text { - Face to face classes and } \\
\text { sessions. } \\
\text { - Location not clear. }\end{array}$ \\
\hline
\end{tabular}




\begin{tabular}{|c|c|c|c|c|c|c|c|}
\hline $\begin{array}{l}\text { Frequency, } \\
\text { duration, } \\
\text { intensity and } \\
\text { fidelity of } \\
\text { implementation }\end{array}$ & $\begin{array}{l}\text { - Telephone calls conducted at } \\
1 \text { and } 4 \text { weeks for all } \\
\text { participants. } \\
\text { - No monitoring of the fidelity } \\
\text { of these contacts was } \\
\text { undertaken. } \\
\text { - Follow-up assessment at } 6 \\
\text { weeks. }\end{array}$ & $\begin{array}{l}\text { - Telephone calls conducted } \\
\text { at } 1,4 \text { and } 9 \text { weeks. } \\
\text { - Hospital or home-based } \\
\text { follow-up at } 12 \text { weeks. }\end{array}$ & $\begin{array}{l}\text { - Call frequency as initiated } \\
\text { by the trained healthcare } \\
\text { professional was weekly } \\
\text { for the first } 2 \text { months, } \\
\text { biweekly during the third } \\
\text { month, and monthly for the } \\
\text { past } 3 \text { months. } \\
\text { - The intervention content, } \\
\text { including topics addressed } \\
\text { and interventions provided } \\
\text { during each WE CALL } \\
\text { contact, was documented } \\
\text { by the trained healthcare } \\
\text { professional. } \\
\text { - The duration of each } \\
\text { telephone contact and the } \\
\text { total number of contacts } \\
\text { were recorded. }\end{array}$ & $\begin{array}{l}\text { - 60-minute initial 1:1 } \\
\text { session, in which the } \\
\text { Nurse Educator and Peer } \\
\text { Dyad met with the } \\
\text { stroke/TIA survivor } \\
\text { - Four } 60 \text {-minute group } \\
\text { sessions with } 6-7 \text { AA } \\
\text { stroke/TIA survivors } \\
\text { - Sessions } 2,3 \text { and } 4 \text { were } \\
\text { held approximately } 15,30, \\
\text { and } 60 \text { days after the initial } \\
\text { session. } \\
\text { - Fidelity was assessed at } \\
\text { each session by non- } \\
\text { interventionist study staff } \\
\text { both quantitatively and } \\
\text { qualitatively. } \\
\text { - Non-interventionist study } \\
\text { staff assessed each TEAM } \\
\text { group with each fidelity } \\
\text { dimension being rated on } \\
\text { a } 1-10 \text { scale. }\end{array}$ & $\begin{array}{l}\text { This programme was } \\
\text { scheduled three times per } \\
\text { week for } 8 \text { weeks, each } \\
\text { session lasting } 2 \text { hours. }\end{array}$ & $\begin{array}{l}\text { The intervention was } \\
\text { delivered to small groups } \\
\text { of } 6-7 \text { participants at a } \\
\text { time across } 12 \text { sessions. }\end{array}$ & $\begin{array}{l}\text { - CDSMP participants were } \\
\text { scheduled to complete the } \\
\text { intervention at the next } \\
\text { available group session, } \\
\text { typically scheduled at the } \\
\text { start of the next calendar } \\
\text { month following baseline } \\
\text { testing. } \\
\text { - The intervention sessions } \\
\text { were two hours long, once } \\
\text { a week, for six weeks. }\end{array}$ \\
\hline $\begin{array}{l}\text { Tailoring and } \\
\text { modifications }\end{array}$ & Not reported. & $\begin{array}{l}\text { Intervention goals were } \\
\text { individually tailored. }\end{array}$ & $\begin{array}{l}\text { - If the participant expressed } \\
\text { concerns about specific } \\
\text { area, the content of the } \\
\text { discussion was noted, and } \\
\text { any intervention provided. } \\
\text { - Additional written } \\
\text { information on stroke } \\
\text { management was provided } \\
\text { as needed (by regular } \\
\text { mail, e-mail, or Internet). }\end{array}$ & Not reported. & Not reported. & Not reported. & Not reported. \\
\hline $\begin{array}{l}\text { Comparator } \\
\text { interventions }\end{array}$ & $\begin{array}{l}\text { Current standard post- } \\
\text { TIA/minor stroke care as per } \\
\text { current UK guidelines, with GP } \\
\text { phone call to discuss their care } \\
\text { at } 1 \text { and } 4 \text { weeks. }\end{array}$ & $\begin{array}{l}\text { Current standard post- } \\
\text { TIA/minor stroke care as per } \\
\text { current UK guidelines, with } \\
\text { GP phone call to discuss } \\
\text { their care at } 1,4 \text { and } 9 \\
\text { weeks. }\end{array}$ & $\begin{array}{l}\text { YOU CALL - participants } \\
\text { provided with the name and } \\
\text { phone number of THCP to } \\
\text { contact at any time. } \\
\text { THCP was told not to probe } \\
\text { for further information but to } \\
\text { answer queries initiated by } \\
\text { participant. }\end{array}$ & $\begin{array}{l}\text { Treatment as usual (TAU) - } \\
\text { Individuals in TAU continued } \\
\text { treatment with their regular } \\
\text { medical care providers. }\end{array}$ & Normal care group. & $\begin{array}{l}12 \text { week waiting period, no } \\
\text { active intervention. Following } \\
12 \text { week wait, received } \\
\text { IPASS intervention. }\end{array}$ & $\begin{array}{l}\text { Post-discharge rehabilitation } \\
\text { services recommended by } \\
\text { treating physician. } \\
\text { Offered CDSMP after they } \\
\text { had completed the study. }\end{array}$ \\
\hline
\end{tabular}


Table 4. Outcomes reported by included studies.

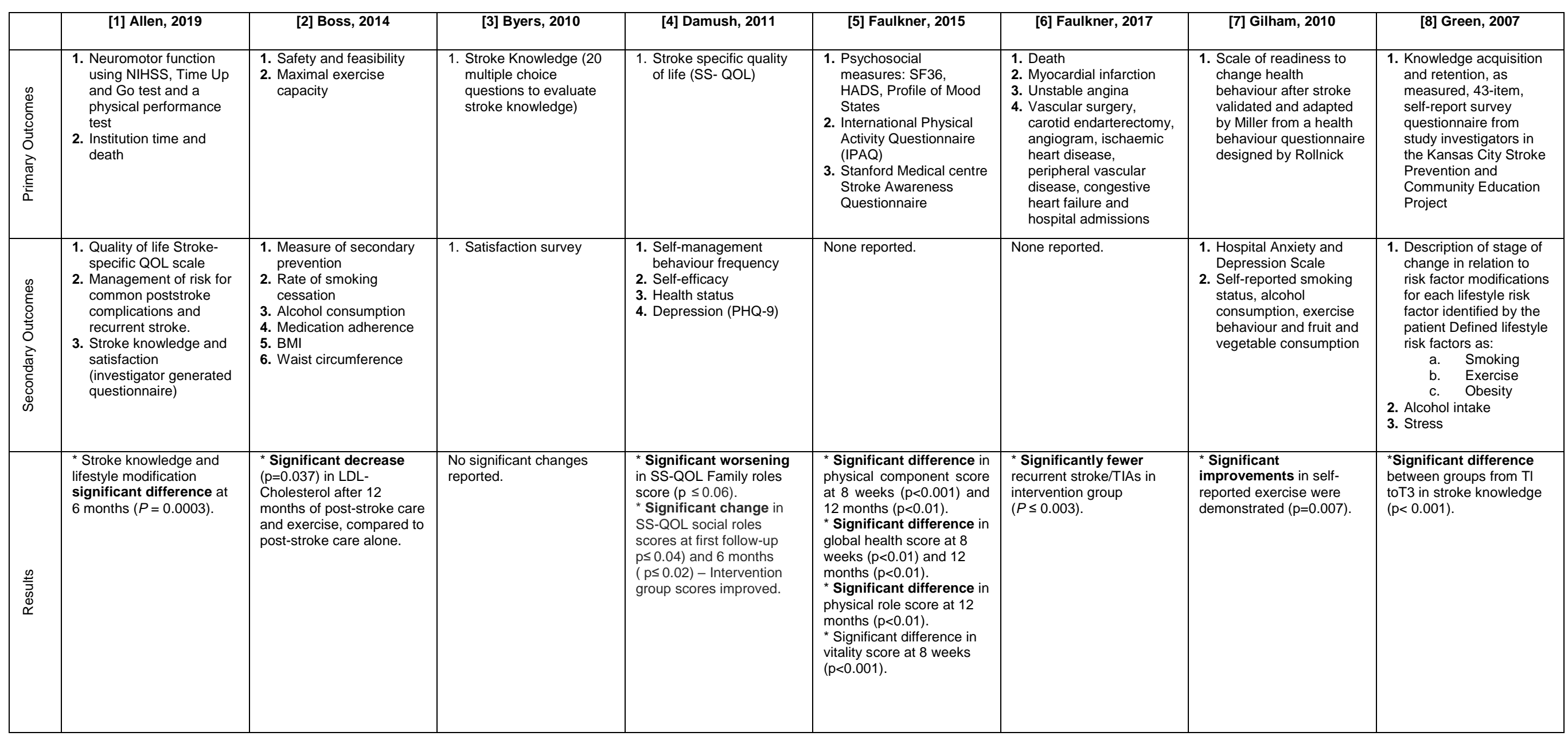


Table 4. Continued.

\begin{tabular}{|c|c|c|c|c|c|c|c|}
\hline & [9] Heron, 2017 & [10] Herron, 2019 & [11] Rochette, 2013 & [12] Sajatovic, 2018 & [13] Wang, 2013 & [14] Wolf, 2016 & [15] Wolf, 2017 \\
\hline 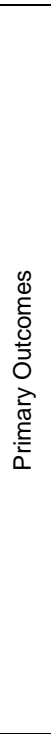 & $\begin{array}{l}\text { 1. Physical activity levels } \\
\text { (validated International } \\
\text { Physical Activity } \\
\text { Questionnaire (IPAQ) } \\
\text { questionnaire } \\
\text { 2. Mediterranean Diet Score } \\
\text { using a validated } \\
\text { questionnaire } \\
\text { 3. } 2 \text { min walk test } \\
\text { 4. Hospital Anxiety and } \\
\text { Depression (HADs) } \\
\text { questionnaire } \\
\text { 5. EQ5D-5 L questionnaire, } \\
\text { quality of life, } \\
\text { 6. Disability - Modified Rankin } \\
\text { scale } \\
\text { 7. Prochaska stages of } \\
\text { change questionnaire } \\
\text { relating to physical activity }\end{array}$ & $\begin{array}{l}\text { 1. Rates of recruitment, } \\
\text { retention and completion of } \\
\text { outcome measures } \\
\text { 2. Height and weight (light } \\
\text { clothing; Seca scale, model } \\
\text { 799) } \\
\text { 3. Waist circumference, } \\
\text { 4. Resting blood pressure and } \\
\text { heart rate) } \\
\text { 5. Physical activity (validated } \\
\text { International Physical } \\
\text { Activity Questionnaire } \\
\text { (IPAQ)) } \\
\text { 6. Mediterranean Diet Score } \\
\text { using a validated } \\
\text { questionnaire. } \\
\text { 7. Anxiety and depression } \\
\text { (Hospital Anxiety and } \\
\text { Depression (HADs) } \\
\text { questionnaire) } \\
\text { 8. Disability (Modified Rankin } \\
\text { scale) } \\
\text { 9. Readiness to change } \\
\text { 10. Quality of life (EQ5D-5L) } \\
\text { 11. Physical activity using } \\
\text { pedometer }\end{array}$ & $\begin{array}{l}\text { 1. Unplanned use of health } \\
\text { services for an adverse } \\
\text { event - Collected daily } \\
\text { through a frequency } \\
\text { calendar } \\
\text { 2. Quality of Life - Quality of } \\
\text { Life Index \& EuroQol 5D }\end{array}$ & $\begin{array}{l}\text { 1. Self-reported medication } \\
\text { treatment adherence with } \\
\text { stroke risk-reduction } \\
\text { pharmacotherapies - } \\
\text { Tablets Routines } \\
\text { Questionnaire (TRQ) }\end{array}$ & $\begin{array}{l}\text { 1. Knowledge of stroke- } \\
\text { stroke warning signs and } \\
\text { medical treatment, risk } \\
\text { factors of stroke and } \\
\text { dietary knowledge } \\
\text { 2. Behaviour - five-point } \\
\text { Likert scale questions. } \\
\text { Warning signs of stroke, } \\
\text { behaviours related to the } \\
\text { risk factors of stroke and } \\
\text { social participation } \\
\text { 3. Self-efficacy towards stroke } \\
\text { prevention including dietary } \\
\text { control, continuous } \\
\text { rehabilitation, physical } \\
\text { activities, exercise, positive } \\
\text { attitude in social } \\
\text { participation, stress } \\
\text { management }\end{array}$ & $\begin{array}{l}\text { 1. Chronic disease -Chronic } \\
\text { disease self-efficacy scale } \\
\text { (CDSES) } \\
\text { 2. Participation strategies - } \\
\text { Self-efficacy scale (PS- } \\
\text { SES) }\end{array}$ & $\begin{array}{l}\text { 1. Health Behaviours - Chroni } \\
\text { c Disease Self- Efficacy } \\
\text { Scale (CDSES) } \\
\text { 2. Health status -Adapted } \\
\text { illness Intrusiveness } \\
\text { Ratings } \\
\text { 3. Healthcare Utilization } \\
\text { - Health Care Utilization } \\
\text { Survey }\end{array}$ \\
\hline 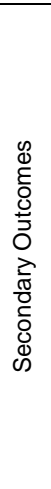 & None reported. & None reported. & $\begin{array}{l}\text { 1. Planned use of health } \\
\text { services - Collected } \\
\text { through frequency calendar } \\
\text { 2. Depression - Beck } \\
\text { Depression Inventory II } \\
\text { (BDI-II) } \\
\text { 3. Participation - Assessment } \\
\text { of Life Habits (LIFE-H 3.1) }\end{array}$ & $\begin{array}{l}\text { 1. Biologic parameters of } \\
\text { stroke risk - measured by } \\
\text { serum HbA1c, serum } \\
\text { cholesterol and } \\
\text { triglycerides, body mass } \\
\text { index/BMI) } \\
\text { 2. Diet - Diet Habit Survey } \\
\text { (DHS) } \\
\text { 3. Exercise - International } \\
\text { Physical Activity } \\
\text { Questionnaire (IPAQ) short } \\
\text { form } \\
\text { 4. Smoking - Fagerstrom } \\
\text { Test for Nicotine } \\
\text { Dependence (FTND) } \\
\text { 5. Substance use - Addiction } \\
\text { Severity Index (ASI) }\end{array}$ & None reported. & $\begin{array}{l}\text { 1. Community participation - } \\
\text { Community participation } \\
\text { indicators (CPI) } \\
\text { 2. Reintegration - } \\
\text { Reintegration to normal } \\
\text { living (RNL) } \\
\text { 3. Activity participation - } \\
\text { Activity card sort (ACS) } \\
\text { 4. Quality of life -WHO quality } \\
\text { of life scale (WHOQOL- } \\
\text { BREF) } \\
\text { 5. Stroke impact -Stroke } \\
\text { impact scale (SIS) }\end{array}$ & $\begin{array}{l}\text { 1. Quality of life - } \\
\text { World Health Organization } \\
\text { Quality of Life Questionnaire } \\
\text { (WHOQOL-BREF) }\end{array}$ \\
\hline 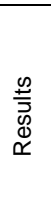 & $\begin{array}{l}\text { No statistical comparison data } \\
\text { reported. }\end{array}$ & $\begin{array}{l}\text { No significant difference in } \\
\text { outcomes. }\end{array}$ & $\begin{array}{l}\text { * Significant change for both } \\
\text { groups from } 6 \text { months to } 1 \\
\text { year }(n=139) \text { in social } \\
\text { domains of the LIFE-H ( } 95 \% \\
\text { confidence interval, } 0.1-0.7 \text {; } \\
\text { effect size, 0.3). }\end{array}$ & $\begin{array}{l}\text { No significant difference in } \\
\text { outcomes. }\end{array}$ & $\begin{array}{l}\text { No significant difference in } \\
\text { outcomes. }\end{array}$ & $\begin{array}{l}{ }^{*} \text { Significant short-term } \\
\text { increase in health-related } \\
\text { self-efficacy both within-group } \\
\text { and between-groups }(p< \\
0.05) \text { in managing chronic } \\
\text { conditions which were } \\
\text { retained at follow-up. }\end{array}$ & $\begin{array}{l}\text { No significant differences } \\
\text { between groups in } \\
\text { demographics or baseline } \\
\text { data with the exception of how } \\
\text { participants felt they are able } \\
\text { to manage their health in } \\
\text { general }(p=0.05) \text {. }\end{array}$ \\
\hline
\end{tabular}

